\title{
Electron Heat Transport in ASDEX Upgrade: Experiment and Modeling
}

\author{
F. Ryter, G. Tardini, H.-U. Fahrbach, K. Kirov, F. Leuterer, A.G. Peeters, \\ G. Pereverzev, W. Suttrop and ASDEX Upgrade Team \\ Max-Planck-Institut für Plasmaphysik, EURATOM Association, \\ D-85748 Garching e-mail: ryter@ipp.mpg.de
}

\section{Electron heat transport driven by turbulence with $\nabla T_{e} / T_{e}$ threshold}

Studies of electron heat transport in ASDEX Upgrade [1, 2] and other tokamaks [3, 4] suggest that transport is governed by turbulence increasing above a threshold $\left(\nabla T_{e} / T_{e}\right)_{c}=1 / L_{T c}$, named $\kappa$ in the following. As a consequence the temperature profiles react weakly to changes of the heating power intensity and deposition profile: "profile resilience" of "stiffness".

Based on these observations a simple analytical transport model has been developed and tested on ASDEX Upgrade data [5]. It is based on the following assumption for the heat diffusivity:

$$
\chi_{e}=\chi_{0}+\lambda T_{e}^{\alpha}\left(\nabla T_{e} / T_{e}-\kappa\right)^{\beta} H_{\kappa}
$$

where $\lambda, \alpha$ and $\beta$ are coefficients to be adjusted, $H_{\kappa}$ is the Heavyside function equals to zero for $\nabla T_{e} / T_{e}<\kappa$ and to unity for $\nabla T_{e} / T_{e} \geq \kappa$. We will show below that good results are obtained with $\alpha=0.5$ and $\beta=1$. In the remaining of this paper, the units are mks except keV instead of $\mathrm{eV}$ for the temperatures. The usual definition of the perturbative heat diffusivity, which defines the propagation of heat pulses, is [6]:

$$
\chi_{e}^{H P}=\chi_{e}^{P B}+\frac{\partial \chi_{e}}{\partial \nabla T_{e}} \nabla T_{e}
$$

with $\chi_{e}^{P B}$ being the power balance or steady-state heat diffusivity. This expression can be derived explicitly from Eq. 1 , giving with $\beta=1$ :

$$
\chi_{e}^{H P}=\chi_{0}+\lambda T_{e}^{\alpha}\left(2 \nabla T_{e} / T_{e}-\kappa\right) H_{\kappa}
$$

under the assumption that $\chi_{0}$ does not depends on $\nabla T_{e}$. This expression shows the important property that $\chi_{e}^{H P}$ increases in a step largely above $\chi_{0}$ as soon as $\nabla T_{e} / T_{e}$ is larger than $\kappa$, whereas $\chi_{e}^{P B}$ increases continuously with $\nabla T_{e} / T_{e}-\kappa$, see [5] and also Fig. 1 right part. The physics validity of the empirical model is supported by the good results obtained in ASDEX Upgrade using the Weiland model for NBI heated plasmas [7] and in ECH heated plasma dominated by electron transport [8]. This first principle model is based on Ion Temperature Gradient for the ions and Trapped Electron Modes driven turbulence for the electrons. These two instabilities both have a respective threshold in $\nabla T / T$. The Electron Temperature Gradient driven turbulence also has a (different) threshold in $\nabla T_{e} / T_{e}$, [9].

As a consequence of this transport property, the temperature profiles exhibit very similar values of $R / L_{T}$ in several tokamaks [3]. Equivalently, this is reflected in each device by the fact that plotted on a logarithmic scale the $T_{e}$ profiles have the same shape and are shifted according to the edge or pedestal temperature, which is therefore a key parameter.

\section{Variation of heat flux at constant edge flux: steady-state and modulation}

The ASDEX Upgrade tokamak is equipped with a flexible ECH system composed of 4 beams whose deposition can be varied independently by mirror launchers. The electron temperature, essential here, is provided by 2 diagnostics: the 60 channel ECE heterodyne radiometer with a spatial resolution of about $1 \mathrm{~cm}$ and a bandwidth of $\approx 31 \mathrm{kHz}$; the Thomson scattering with 16 radial channels and a profile every $16 \mathrm{~ms}$. These two diagnostics agree within $\pm 10 \%$. The 

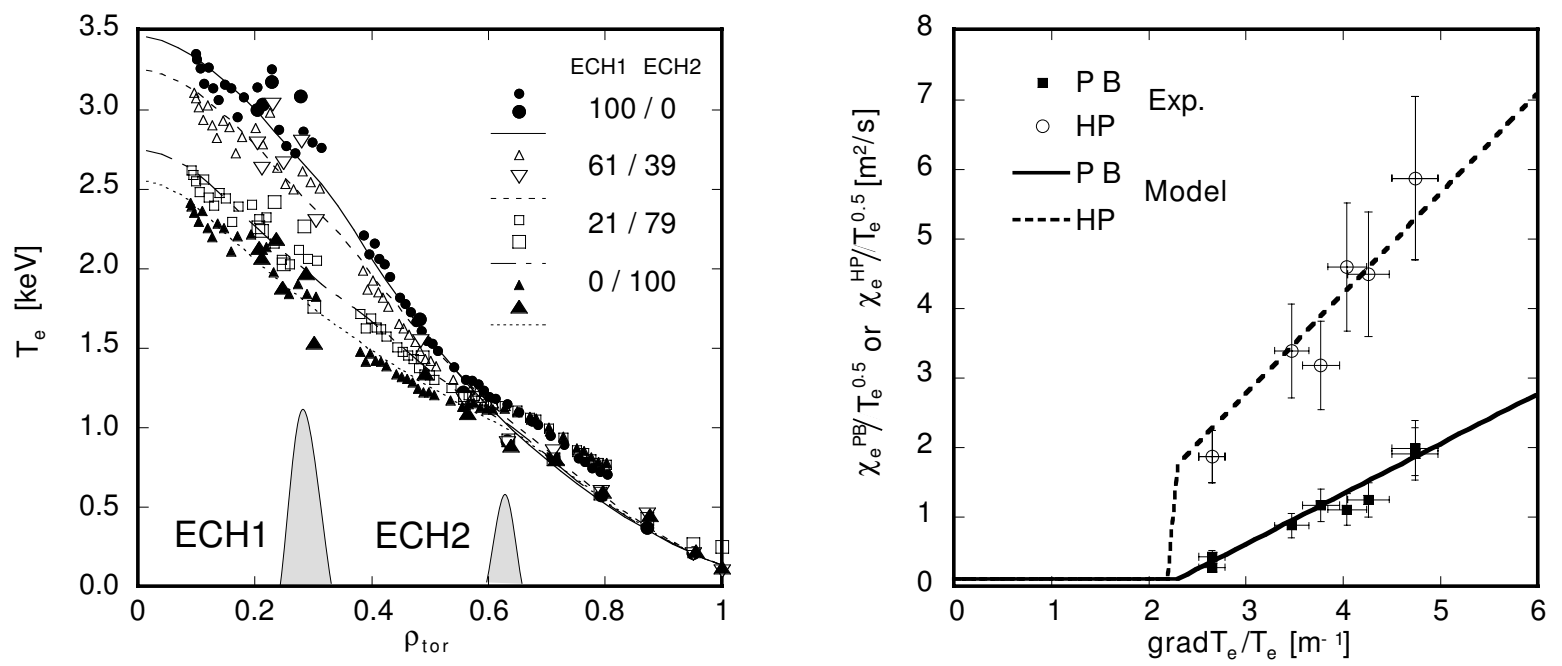

Figure 1: Left: $T_{e}$ profiles in the experiments with heat flux variation by ECH in the confinement region with constant edge flux $\left(P_{E C H 1}+P_{E C H 2}=1.3 M W\right)$. Small symbols for ECE, large symbols for Thomson scattering, lines for modelling. Right: Results from power balance and heat pulse analysis at $\rho_{t} \approx 0.5$. The lines are given by the model from $E q .1$ with $\lambda=0.72$ and $\kappa=2.3$

other quantities are provided by the usual diagnostics available on a modern tokamak.

According to the considerations of Sect. 1, it is essential in transport studies to vary the heat flux in the confi nement region at constant edge temperature. This was achieved in new experiments where we varied the electron heat flux in the confi nement region $\left(0.35 \leq \rho_{t} \leq\right.$ $0.7)$ by one order of magnitude while keeping the heat flux at the plasma edge $\left(\rho_{t} \geq 0.65\right)$ constant. For this purpose, we deposited the ECH power at $\rho_{1} \approx 0.35$ and $\rho_{2} \approx 0.65$ with the respective intensities $P_{E C H 1}$ and $P_{E C H 2}$. These were varied while keeping $P_{E C H 1}+P_{E C H 2}$ constant at about 1.3 MW. The discharges were run in deuterium at $I_{p}=800 \mathrm{kA}, B_{T}=2.3$ $\mathrm{T}$, and $\bar{n}_{e}=210^{19} \mathrm{~m}^{-3}$ to reduce the electron-ion energy transfer and provide good conditions to study the electron heat transport. In addition, modulation of $P_{E C H 1}$ or $P_{E C H 2}$ allows to compare transient transport $\left(\chi_{e}^{H P}\right)$ to power balance $\left(\chi_{e}^{P B}\right)$. In both experiment and modelling the Fourier transform of the modulated $T_{e}$ data yields profi les of amplitude and phase at the frequency of the power modulation for a quantitative comparison. The values of $\chi_{e}^{H P}$ are deduced from these profi les by the usual techniques described in $[6,1]$.

The steady-state temperature profi les are shown in Fig. 1 left part for a selection of these discharges. Indeed, under these conditions a clear variation of $\nabla T_{e}$ and $\nabla T_{e} / T_{e}$ can be achieved , which is not the case when central heating only is varied because the edge temperature increases with heating power. Note here the reproducibility of the edge profi les for $\rho_{t} \geq 0.65$. The results of power balance and transient transport $\rho_{t} \approx 0.5$ are shown in Fig. 1 right part. There, the $\chi$ values are divided by the $T_{e}^{1 / 2}$ dependence of the model to correct for the (moderate) variation of $T_{e}$ in the region of analysis. A linear fi $t$ through the power balance data, neglecting the very small contribution from $\chi_{0}$, yields $\lambda=0.72$ and $\kappa=2.3$ (solid line in the figure). According to Eq. 3 we can also calculate the corresponding values for transient transport. The result, dashed line in Fig. 1 right part, agrees well with the experimental data. These values of $\lambda$ and $\kappa$ were then taken for transport simulations with the ASTRA code using Eq. 1. The results, lines in Fig. 1 left part, agree very well with the experimental data over the whole radius. The boundary condition is the temperature at $\rho_{t}=1.0$. It must be mentioned 

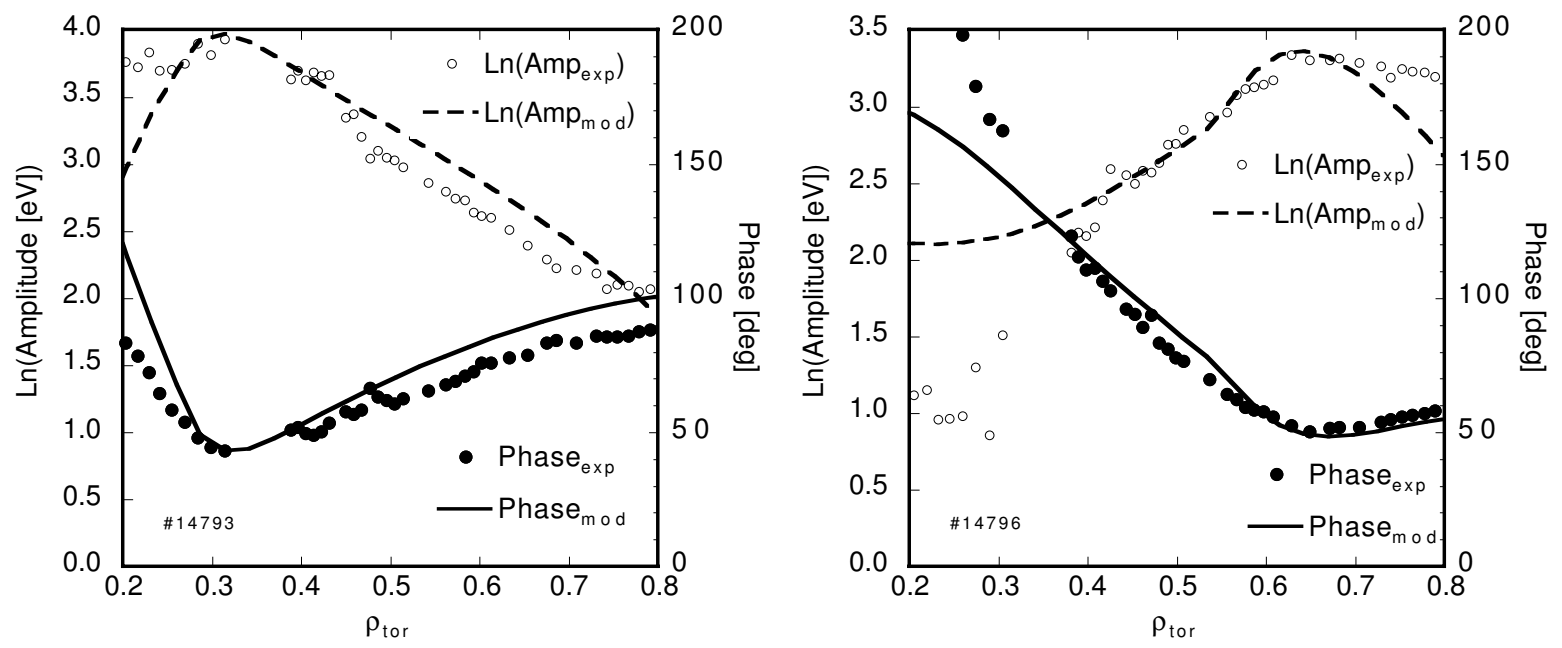

Figure 2: Amplitude and phase of the $T_{e}$ modulation for the two extreme cases of Fig. $1, P_{E C H 1}=100 \%$ (left) or $P_{E C H 2}=100 \%$ (right). The points are the data, the lines the empirical model model, both with the same values $\lambda=0.72$ and $\kappa=2.3$.

that dividing the $\chi$ values by $T_{e}^{3 / 2}$, as taken in [5], instead of $T_{e}^{1 / 2}$ yields values which are lower by about a factor of 2 for $\lambda$ and by about $30 \%$ for $\kappa$. With these values the simulations yield quite poor agreement.

The simulations also include the power modulation made in the experiment. The results of the Fourier transform of the experimental and modelled $T_{e}$ are illustrated in Fig. 2 by the two extreme cases with central or edge heating. The agreement is quite satisfactory. The intermediate cases give comparably good results. It must be underlined that under theses experimental conditions with the pure off-axis heating the $T_{e}$ profi les inside the ECH2 deposition is just above but close to the threshold $\kappa$. Indeed, in such off-axis cases, $\chi_{e}^{P B}$ is very low, but , as expected from the model and also shown by Fig. $1 \chi_{e}^{H P}$ stays rather high. We also observed experimentally and in the model that, as expected, this ratio goes down to about unity, with very low values of $\approx 0.2 \mathrm{~m}^{2} / \mathrm{s}$ for both $\chi_{e}^{P B}$ and $\chi_{e}^{H P}$, when the $T_{e}$ profi le drops below the threshold. It must be emphasized that the rapid variation of $\chi_{e}^{H P}$ for very small changes of $\chi_{e}^{P B}$ and $\nabla T_{e} / T_{e}$ is a monitor of the status of the $T_{e}$ profi le: above or below the threshold. Therefore, such conditions deliver a direct measurement of the threshold and might allow to discriminate between physics hypotheses on the turbulence involved. In particular, the good results obtained using $\kappa$ without dependence on radius or plasma parameters suggest that the actual threshold should also be quite insensitive to plasma values. This is the case for the TEM driven turbulence [10], not for the ETG turbulence for which the threshold depends strongly on $T_{e} / T_{i}$ and $s / q$ [9].

As already found for others discharges with similar conditions the ITG/TEM Weiland model [10] gives for both the steady-sate and modulation data quite good results [8], which are very similar to those obtained with the empirical model.

\section{Variation of plasma current}

We compare now two L-mode discharges at $B_{T}=2.3 \mathrm{~T}, 800 \mathrm{kA}\left(q_{95}=4.8\right.$, from Sect. 2) and $400 \mathrm{kA}\left(q_{95}=9.8\right)$, in their Ohmic phase and in their phase heated almost centrally $\left(\rho_{t} \approx 0.35\right)$ by $1.3 \mathrm{MW}$ of ECH. The discharge at $800 \mathrm{kA}$ is sawtoothing, the inversion radius being $\rho_{t} \approx 0.25$ whereas that at $400 \mathrm{kA}$ is sawtooth-free due to the high $q_{95}$ value. The density 

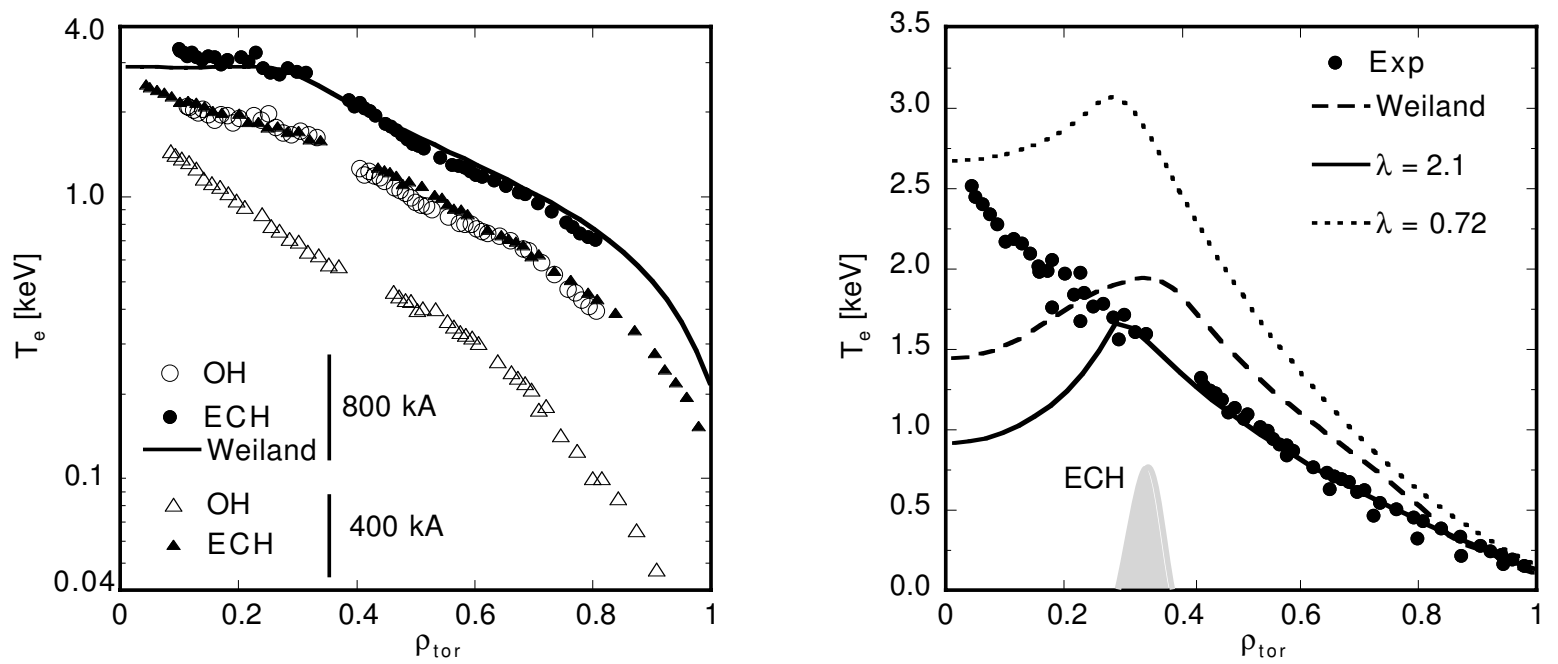

Figure 3: Experimental and modelled $T_{e}$ profi les for L-mode plasmas with $1.3 \mathrm{MW}$ ECH central deposition. Left: Experiment at $I_{p}=800 \mathrm{kA}$ and $400 \mathrm{kA}$, and comparison with the Weiland model at $800 \mathrm{kA}$ (log scale). Right: Data at $400 \mathrm{kA}$ and models as indicated in the legend and text.

is kept low $\left(\bar{n}_{e}=210^{19} \mathrm{~m}^{-3}\right)$ to reduce the ion-electron energy exchange. The temperature profi les are shown in Fig. 3. We do not consider the part of the plasma inside the ECH deposition where the electron heat flux is very low which makes modelling or power balance very delicate, and where in addition sawteeth mainly determine the profi le at $800 \mathrm{kA}$.

The data in the left plot (log scale) show the very similar slope of the profi les over a major part of the core plasma, as mentioned in Sect. 1. In this region $\nabla T_{e} / T_{e}$ is almost constant and the profi les are shifted, depending on the temperature in the edge region. The Ohmic case at $400 \mathrm{kA}$ seems to behave differently, which might be attributed to the very low temperature, low electron heat flux and strong losses to the ions. Note the almost perfect coincidence of the profi les with $\mathrm{ECH}$ at $400 \mathrm{kA}$ and Ohmic at $800 \mathrm{kA}$. The Weiland model agrees well with the $\mathrm{ECH}$ shot at $800 \mathrm{kA}$, as also shown in [8]. In the right part of the fi gure we compare the profi le with ECH at $400 \mathrm{kA}$ with the models. For the empirical model (Eq. 1) we could keep $\kappa=2.3$. However the value of $\lambda$ must be different for the two values of current: $\lambda=0.72$ at $800 \mathrm{kA}$ and $\lambda=2.1$ at $400 \mathrm{kA}$. Note the perfect agreement with $\lambda=2.1$ and the strong disagreement with $\lambda=0.72$. This means that transport is much higher at $400 \mathrm{kA}$, in agreement with the usual $I_{p}$ dependence. The Weiland model does not perform well at $400 \mathrm{kA}$, as shown in Fig 3 right, even forcing the temperature to take the experimental value at $\rho_{t} \approx 0.8$.

\section{References}

[1] RYTER, F. et al., Phys. Rev. Lett. 86 (2001) 2325.

[2] RYTER, F. et al., Phys. Rev. Lett. 86 (2001) 5498.

[3] RYTER, F. et al. Plasma Phys. Contr. Fusion 44 (2002) A407.

[4] HOANG, G. T. et al., Phys. Rev. Lett. 87 (2001) 125001.

[5] IMBEAUX, F. et al., Plasma Phys. Controlled Fusion 43 (2001) 1503.

[6] LOPES CARDOZO, N. J., Plasma Phys. Controlled Fusion 37 (1995) 799.

[7] TARDINI, G. et al., Nucl. Fusion 42 (2002) 258.

[8] TARDINI, G. et al., Nucl. Fusion 40 (2002), in press.

[9] JENKO, F. et al., Phys. Plasmas 8 (2001) 4096.

[10] NORDMAN, H. et al., Nucl. Fusion 30 (1990) 983. 